Apidologie, 1978, 9 (4), 349-362.

\title{
EINFLUSS DER - IN EINEM FLUGRAUM KONTROLLIERTEN - TRACHTVERHALTNISSE AUF DAS DUFTMARKIEREN UND DIE ABFLUGMAGENFULLUNG DER SAMMELBIENE
}

\author{
Influence des conditions de miellée - contrôlées en chambre \\ de vol - sur le marquage olfactif et le remplissage \\ du jabot d'une abeille butineuse \\ Walter PFLUMM, Cornelia PESCHKE $\uparrow$, \\ Karl WILHELM und Holk CRUSE (*) \\ Fachbereich Biologie der Universität, Postfach 3049, D-6750 Kaiserslautern
}

\begin{abstract}
SUMMARY
INFLUENCE OF THE STATE OF THE GENERALIZED FOOD SUPPLY WHICH IS CONTROLLED IN A BEE ROOM ON THE SCENT MARKING BEHAVIOUR AND THE VOLUME OF THE LOAD PER TRIP OF A FORAGING BEE ( $A$ PIS MELLIFICA).
\end{abstract}

In a bee room the state of the generalized food supply was varied by offering sucrose solutions of different concentrations to the foragers. The solutions were given in a so called " bee room generalized food source". In a second food source (experimental food source) the concentration of the sucrose solution was kept constant. At this food source only a single bee was allowed to drink at the time, her behaviour was measured as a function of the concentration of the solution in the bee room generalized food source.

The length of time during which the scent organ (Nasonov gland) is everted during drinking (at the experimental food source) shortens with increasing concentration of the solution in the bee room generalized food source (i.e. with improvement of the state of the generalized food supply : Fig. 3). The state of the generalized food supply does not have an influence on the volume of the load per trip and on the rate of drinking, if the concentration of the solution in the bee room generalized food source is lower than that in the experimental food source, or if the solution in both food sources in the same (Figs. 4 and 5).

The parameters measured in the bee room can be compared with those obtained in the open, if the state of the generalized food supply is kept constant and the concentration of the solution in the experimental food source is varied (Fig. 1).

(*) Der tödlich verunglückten Cornelia PeschKe zum Gedächtnis; sie hat die Experimente durchgeführt, welche die Abflugmagenfüllung betreffen. 


\section{ZUS AMMENFASSUNG}

In einem Flugraum wurden variable Trachtverhältnisse dadurch nachgeahmt, dass die Sammlerinnen (Apis mellifica) verschieden konzentrierte Saccharoselösungen in einer Trachtfutterquelle geboten erhielten. In einer zweiten Futterquelle (=Versuchsfutterquelle) wurde die Konzentration der Saccharoselösung konstant gehalten. Hier wurde jeweils nur eine Einzelbiene zugelassen und ihr Verhalten in Abhängigkeit von der Konzentration der Lösung in der Trachtfutterquelle registriert.

Es ergab sich: Mit zunehmender Konzentration der Lösung in der Trachtfutterquelle (d.h. mit Verbesserung der Trachtverhältnisse) verkürzt sich die Dauer des Duftmarkierens während des Saugens (Abb. 3). Spendet die Trachfutterquelle niedriger konzentrierte Lösung als die Versuchsfutterquelle, oder ist die Konzentration der Lösung in beiden Futterquellen die gleiche, werden die Abflugmagenfüllung und die Sauggeschwindigkeit nicht von den Trachtverhältnissen beeinflusst (Abb. 4 und 5).

Bei konstant gehaltenen Trachtverhältnissen und Variation der Konzentration der Lösung in der Versuchsfutterquelle sind die im Flugraum erhaltenen Messgrössen des Verhaltens vergleichbar mit im Freiland gewonnenen (Abb. 1).

Sammelbienen zeigen an einer Futterquelle durch ihr Verhalten, wie sie diese "beurteilen ". Ist das Futterangebot gut, stülpen sie, die Futterquelle markierend, ihr Duftorgan aus - was sie auch in verschiedenen anderen Situationen tun (Literaturübersicht bei RENNER, 1960). Mit zunehmender Konzentration der Zuckerlösung wächst die Dauer dieses Duftmarkierens. Dabei steigt gleichsinnig auch die Füllung ihres Honigmagens, die "Abflugmagenfüllung" (Literaturzusammenstellung bei PrLumm, 1969 a). Während eines Sammelzyklus wird die Biene aber nicht nur von der Reizsituation an der Futterquelle beeinflusst, sondern auch während der Abgabe des Sammelgutes im Stock : So wird ihr - je nach den herrschenden Trachtverhältnissen - eine Lösung gegebener Konzentration verschieden schnell abgenommen (LiNDAUER, 1948). Die Trachtverhältnisse bestimmen also indirekt über das Verhalten der Abnehmerinnen im Stock das der Sammlerin an der Futterquelle: Bei guter Tracht wird eine relativ hoch konzentrierte (z.b. $25 \%$ ige) Lösung nicht eingetragen (v. Frisch, 1965) - wie auch in der praktischen Imkerei bekannt ist. Wie hängen die Dauer des Duftmarkierens, die Abflugmagenfüllung und die Sauggeschwindigkeit von den Trachtverhältnissen ab? Dies lässt sich in einem Flugraum prüfen, wo die gesamten Trachtverhältnisse experimentell variiert werden können. Versuche hierzu sind im folgenden geschildert (ergänzt durch Vergleichsmessungen im Freiland).

\section{METHODIK}

Unter Trachtverhältnissen wird hier in Übereinstimmung mit v. FRISCH (1935, 1965), LiNDAUER (1948) und BOCH (1956) das Nektar-Angebot sämtlicher Futterquellen verstanden, die von den Stockgenossinnen der Versuchsbiene beflogen werden.

a) Experimente im Flugraum: Die Versuche wurden von August bis November 1975 und 1976 mit je einem Volk der Krainer Rasse durchgeführt. Der Stock stand bis zum Beginn der einwöchigen Eingewöhnungszeit im Freien. Der Flugraum $\left(5,9 \times 3,4 \times 3,0 \mathrm{~m}\right.$; Temperatur $\left.23 \pm 3{ }^{\circ} \mathrm{C}\right)$ nach Renner 
(1955) und van PraAgh (1974) war mit UV-Leuchtstoffröhren Philips TL A 65-80 W/05 versehen (Beleuchtungsstärke $1 \mathrm{~m}$ über dem Boden $220 \mathrm{Lux}$; Beleuchtungsdauer von 7.45 bis $20.45 \mathrm{Uhr}$ ). Die relative Luftfeuchte schwankte zwischen 50 und $60 \%$. Wasser stand ad lib. zur Verfügung, alle $11 / 4$ Stunden wurde frischer Pollen (gemahlene Höschen) angeboten.

Zuckerwasser gab es in $3 \mathrm{~m}$ Entfernung vom Stock in einem (pneumatischen) Futtergefäss; dieses war eine abgewandelte Form des Rillengefässes nach RENNER (1959): Das Vorratsgefäss stand so in einem Schälchen (Form einer Petrischale; Durchmesser 5,5 cm; Höhe $7 \mathrm{~mm}$ ), dass sich statt der Rillen um den unteren Rand des Vorratsgefässes eine $7 \mathrm{~mm}$ breite Rinne zog, in der die Lösung etwa $5 \mathrm{~mm}$ hoch stand; " voller Zufluss " der Lösung (vgl. hierzu Pflumm, 1969 a) war damit gewährleistet. Dieses Futtergefäss wird im folgenden als Trachtfutterquelle bezeichnet.

Die Versuchsfutterquelle (künstliche Blume, s. Abb. 1 bei PfLumm, 1977) stand $1 \mathrm{~m}$ von der Trachtfutterquelle entfernt in einem angrenzenden Raum (Temperatur $24 \pm 1,5^{\circ} \mathrm{C}$ ), in den die Sammlerinnen durch eine $40 \times 40 \mathrm{~cm}$ grosse Durchflugöffnung gelangten. Dort wurde mit Einzelbienen experimentiert. Neulinge wurden weggefangen.

Der Wechsel der verschieden konzentrierten $\mathrm{D}-(+)$ Saccharoselösungen geschah in statistischer Zufallsreihenfolge (Konzentrationsangaben in \%, d.h. g pro $100 \mathrm{~g}$ Lösung; Abkürzung für die Konzentration : c) Bei Änderung der Konzentration der Lösung in der Trachtfutterquelle (d.h. bei Variation der "Trachtverhältnisse ") wurde die Konzentration der Lösung in der Versuchsfutterquelle konstant gehalten und umgekehrt.

In einem Sonderversuch (s. A) wurden auch Messungen an den an der Trachtfutterquelle verkehrenden Bienen durchgeführt.

Bei allen Experimenten herrschte voller Zufluss der Lösung, d.h. die Bienen konnten trinken, so schnell sie vermochten.

Die Abflugmagenfüllung wurde auf folgende Weise gemessen (vgl. Pflumm, 1977; Abb. 1): Die Zuckerlösung floss der Sammlerin durch eine Messpipette zu, eine in dieser mitwandernde Luftblase zeigte an der Pipettenskala die gesaugte Menge an.

b) Experimente im Freien (Ergebnisse in der Einschalffigur $A b b .1$ ): In der zweiten Julihälfte 1974 wurden bei einer Temperatur von $19,0 \pm 1,5^{\circ} \mathrm{C}$ mit einer Schar von $20-30$ Bienen Versuche in $6 \mathrm{~m}$ Entfernung vom Stock durchgeführt. Durch Verwenden eines offenen Glasschälchens (kleine Petrischale) als Futterquelle war für alle Sammlerinnen voller Zufluss gewährleistet. Die Lösungen wurden in folgender Reihenfolge angeboten : $23.7: 30 \%, 4 \% ; 24.7: 55 \%, 16 \% ; 26.7: 55 \%, 8 \%, 2 \%, 4 \%, 16 \%, 30 \%$, $4 \%$. Da nicht alle an einem Schälchen saugenden Bienen gleichzeitig beobachtet werden konnten, wurde folgendermassen vorgegangen : Bei einer bestimmten Biene wurde die Zeitdauer vom Saugbeginn bis zum Einstülpen des Duftorgans registriert, die folgende Messung wurde bei der Biene vorgenommen, die als nächste landete.

Da beim Duftmarkieren starke interindividuelle Schwankungen auftreten (vgl. v. Frisch, 1923; Ribbands, 1955; Pflumm, 1969 a; NúÑEZ, 1971), wurde bei den mit Einzelbienen durchgeführten Versuchen bei der Auswertung folgendermassen vorgegangen: Aus den bei einer bestimmten Konzentration erhaltenen Messwerten einer Biene wurde ein Zentralwert errechnet; aus diesem und entsprechenden Zentralwerten anderer Sammlerinnen wurde ein Mittelwert errechnet, dieser ist dargestellt.

Für die statistische Bearbeitung der Daten wurde der Kolmogoroff-Smirnov-Test verwendet, als Signifikanzniveau (wenn nähere Angaben fehlen) $5 \%$ gewählt.

\section{ERGEBNISSE}

\section{A. Vergleich von im Flugraum gewonnenen Messgrössen des Verhaltens mit solchen aus dem Freiland}

Nach RUTTNER und KoenIGER (1977) “ erreichen die Bedingungen im Flugraum hinsichtlich des biologischen Effektes nicht das Niveau der Haltung im Freien ". Daher ist zunächst das Verhaiten im Flugraum mit dem im Freiland zu vergleichen. 
Bei konstant gehaltenen Trachtverhältnissen wurden Verhaltensweisen des Duftmarkierens und die Anzahl der Neulinge in Abhängigkeit von der Konzentration der Lösung in der Versuchsfutterquelle gemessen (Ergebnisse in Abb. 1).

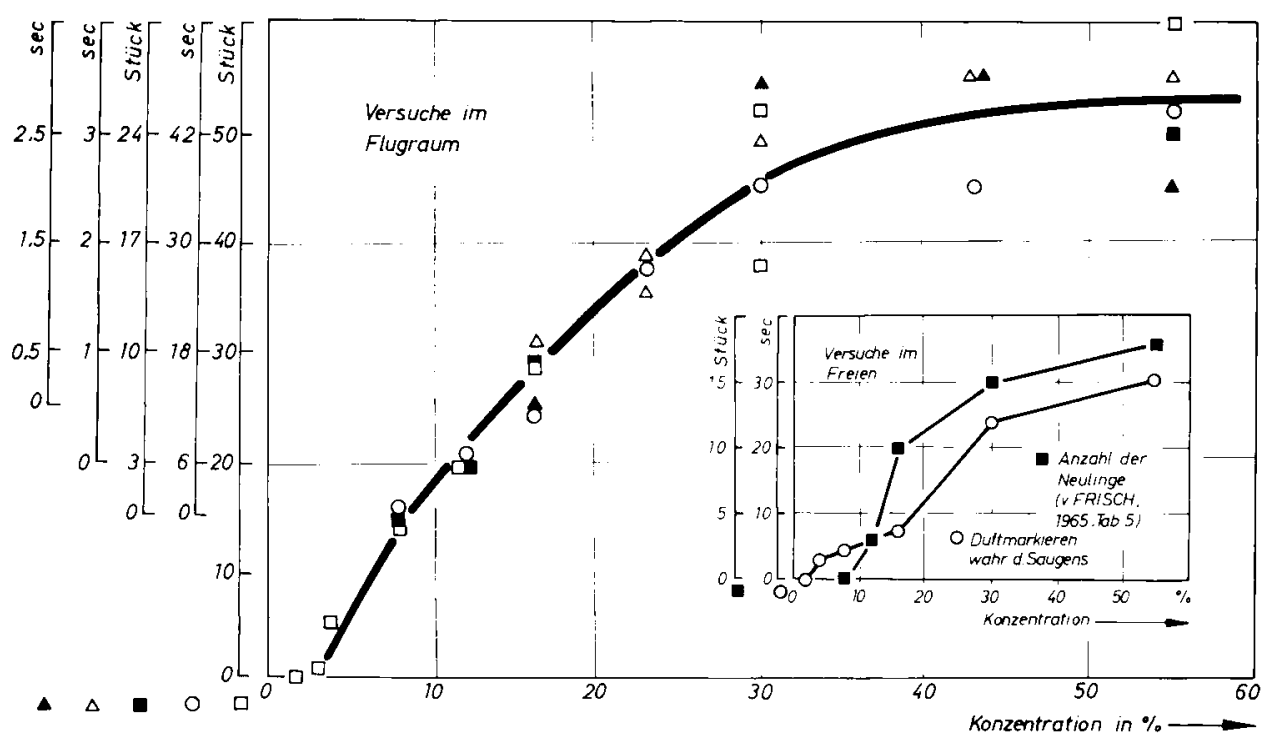
$\triangle$ Daver des Sterzeins vor dem Saugen
- Daver des Sterzeins wahrend des Saugens
- Dauer des Dutrmarkierens wahrend des Saugens
- Anzohl der Neulinge
$\square$ Anzohl der Sommlerinnen

\section{AвB. 1. - Verschiedene Verhaltensweisen und Bienenanzahlen in Abhängigkeit von der Konzentration.}

Versuche im Flugraum. Werte für das Duftmarkieren (Konzentration der Lösung in der Trachtfutterquelle bei diesen Versuchen : $4 \%$ ) : 4 Bienen; Mittelwerte von Zentralwerten. $34<\mathrm{n}<107$. Anzahl der Sammlerinnen (Sonderversuch) : Mittelwerte; $3<\mathrm{n}<5$. Anzahl der Neulinge : Mittelwerte; $6<\mathrm{n}<14$.

Einschaltfigur: Vergleichswerte von Versuchen im Freien. - Duftmarkieren : Juli 1974. 20-30 Bienen. Mittelwerte. $96<\mathrm{n}<251$. Die Unterschiede zwischen folgenden Werten sind signifikant : $55 \%$ $16 \%: \mathrm{P}<0,001 ; 30 \%-16 \%: \mathrm{P}<0,001$.

\section{FIG. 1. - Divers types de comportement et nombres d'abeilles} en relation avec la concentration.

Essais en chambre de vol. Valeurs pour le marquage olfactif (concentration de la solution à la source de nourriture de la chambre de vol pour ces essais : $4 \%$ ): 4 abeilles; moyennes des valeurs médianes. $34<\mathrm{n}<107$. Nombre de butineuses (expérience spéciale) : moyennes : $3<\mathrm{n}<5$. Nombre de novices : moyennes; $6<\mathrm{n}<14$.

Figure encadrée : valeurs pour les essais en plein air. Marquage olfactif : juillet 1974. 20-30 abeilles. Moyennes. $96<\mathrm{n}<251$. Les différences entre les valeurs suivantes sont significatives : $55 \%-16 \%$ : $\mathrm{P}<0,001 ; 30 \%-16 \%: \mathrm{P}<0,001$.

$\triangle$ Durée (en sec.) du rappel avant la prise de nourriture.

$\Delta$ Durée (en sec.) du rappel pendant la prise de nourriture.

Durée (en sec.) du marquage olfactif pendant la prise de nourriture.

- Nombre de novices.

$\square$ Nombre de butineuses. 
In einem Sonderversuch wurde die Konzentration der Lösung in der Versuchsfutterquelle konstant gehalten und die Anzahl der (mit Farbtupfen markierten) Sammlerinnen an der Trachtfutterquelle in Abhängigkeit von der dort gebotenen Konzentration registriert (s. Abb. 1). Bei diesem Sonderversuch wurde die übliche Trachtfutterquelle durch eine offene Plastikschale von $15 \mathrm{~cm}$ Durchmesser und $1 \mathrm{~cm}$ Höhe ersetzt. Damit war für jede Biene voller Zufluss gewährleistet. Erstaunlich ist, dass bei niedriger Konzentration ein Teil der Sammlerinnen darauf verzichtet, diese Lösung einzutragen.

Der Verlauf der Kurve in Abb. 1 entspricht dem der Kurven in der Einschaltfigur Abb. 1, welche Messwerte aus dem Freiland wiedergibt; ähnliche Kurven finden sich in Abb. 5 bei Pflumm (1969 a), welche ebenfalls Daten von Versuchen wiedergibt, bei denen der Stock im Freien stand (zur Art der Darstellung der Messwerte vgl. PflumM, 1969 a).

In Abb. 2 ist die Abflugmagenfüllung als Funktion der Konzentration der Lösung in der Versuchsfutterquelle (bei konstanten Trachtverhältnissen) dargestellt. Diese

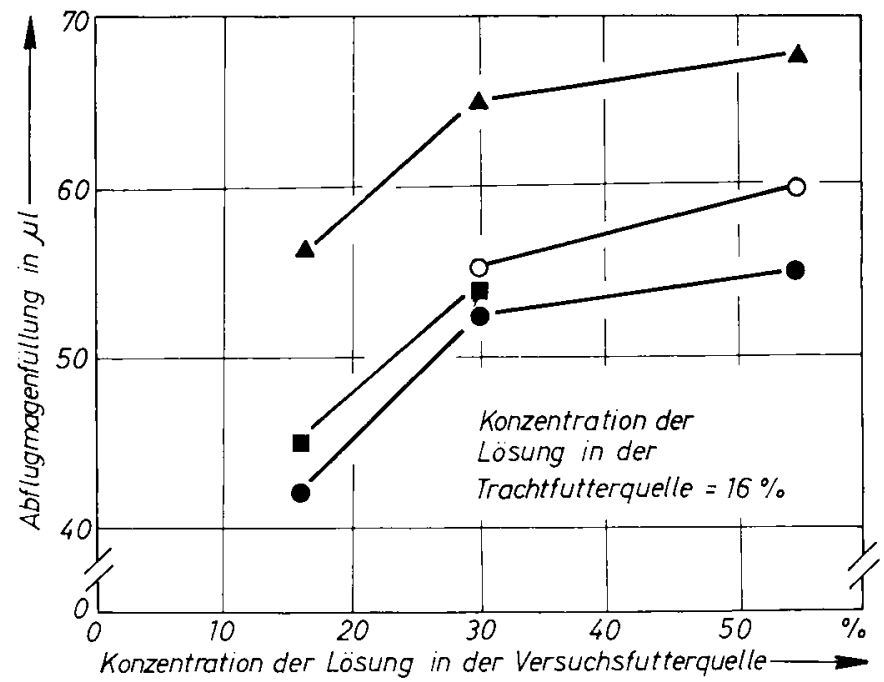

ABв. 2. - Abflugmagenfüllung als Funktion der Konzentration der Lösung in der Versuchsfutterquelle.

Konzentration der Lösung in der Trachtfutterquelle konstant. Jedes Symbol repräsentiert eine Biene. Zentralwerte. $3<n<29$. Für die Differenz zwischen den Werten bei $c=16 \%$ und $c=30 \%$ ergab sich : Geschlossene Kreis- und Quadratsymbole : jeweils $\mathbf{P}<0,01$; geschlossene Dreiecke : $0,01<\mathbf{P}<0,05$. Bei den Werten der durch ein Dreieckssymbol repräsentierten Biene ergab sich ausserdem für den Unterschied zwischen den Werten bei $\mathrm{c}=30 \%$ und $\mathrm{c}=55 \%: \mathrm{P}=0,05$.

FIG. 2. - Remplissage du jabot en fonction de la concentration de la solution à la source expérimentale de nourriture.

Concentration de la solution à la source de nourriture de la chambre de vol : constante $(16 \%)$. Chaque symbole figure une abeille. Valeurs médianes. $3<\mathrm{n}<29$. Les différences entre les valeurs à $c=16 \%$ et $c=30 \%$ sont les suivantes : symboles « rond plein " et "carré plein ": $p<0,01$; symbole " triangle plein " : 0,01<p<0,05. En outre la différence entre les valeurs des abeilles représentées par un triangle à $\mathrm{c}=30 \%$ et $\mathrm{c}=55 \%$ est $\mathrm{P}=0,05$.

En ordonnées : volume du contenu du jabot (en $\mu l$ ).

En abscisses : concentration de la solution à la source expérimentale de nourriture (en \%). 
Messwerte aus dem Flugraum gleichen den im Freien gewonnenen (vgl. z.B. Abb. 2 bei Pflumm, 1977), wo die Trachtverhältnisse vermutlich ebenfalls konstant waren (vgl. PFLUMM, 1969 a, S. 24).

\section{B. Duftmarkieren, Abflugmagenfüllung und Sauggeschwindigkeit in Abhängigkeit von den Trachtverhältnissen}

Mit zunehmender Konzentration der Lösung in der Trachtfutterquelle - bei gleichbleibender Konzentration der Lösung in der Versuchsfutterquelle - nimmt die Dauer des Duftmarkierens an der Versuchsfutterquelle ab (Abb. 3).

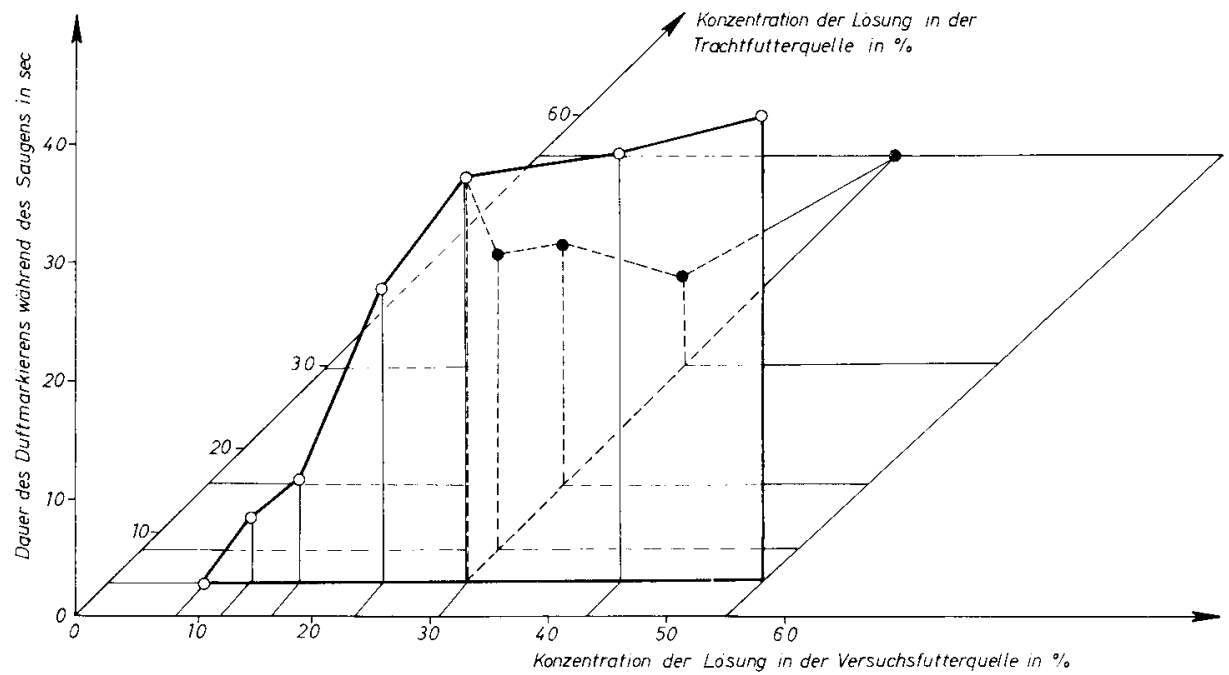

Aвв. 3. - Dauer des Duftmarkierens während des Saugens in Abhängigkeit von der Konzentration der Lösung in der Versuchsfutterquelle und den Trachtverhältnissen (= Konzentration der Lösung in der Trachtfutterquelle).

Durch offene Kreise symbolisierte Werte übernommen aus Abb. 1. Geschlossene Kreissymbole : 2 Bienen. Mittelwerte von Zentralwerten. $24<\mathrm{n}<48$. Sämtliche Werte der Abbildung sind normiert auf den Wert des gemeinsamen Punktes beider Kurven, die normierten Werte wurden anschliessend in Sekunden umgerechnet. Der Wert für den Bezugspunkt wurde dadurch erhalten, dass aus sämtlichen unter diesen Bedingungen gewonnenen Zentralwerten ein gemeinsamer Mittelwert errechnet wurde. Für die durch geschlossene Kreissymbole dargestellten Werte gilt : Differenz zwischen den Werten bei $c=30 \%$ und $c=55 \%$ statistisch gesichert $(P<0,01)$; die Unterschiede zwischen den übrigen Werten sind nicht signifikant.

Fig. 3. - Durée du marquage olfactif au cours de la prise de nourriture en relation avec la concentration de la solution à la source expérimentale de nourriture et celle à la source de nourriture de la chambre de vol.

Les valeurs de la fig. 1 sont représentées par des ronds vides. Ronds pleins : 2 abeilles. Moyennes des valeurs médianes. $24<n<48$. Les valeurs totales de la figure sont normalisées d'après la valeur du point commun aux deux courbes, les valeurs normalisées sont finalement converties en secondes. La valeur du point de référence est obtenue en calculant une moyenne commune à partir des valeurs médianes totales obtenues dans ces conditions. La différence entre les valeurs représentées par des cercles pleins à $\mathrm{c}=30 \%$ et $c=55 \%$ est statistiquement significative $(P<0,01)$; les différences entre les autres valeurs ne sont pas significatives.

Verticalement : durée du marquage olfactif pendant la prise de nourriture, en sec.

Horizontalement : concentration de la solution à la source expérimentale de nourriture, en \%.

En biais : concentration de la solution à la source de nourriture de la chambre de vol, en \%. 
Wenn die Trachtfutterquelle geringer konzentrierte Lösung spendet als die Versuchsfutterquelle oder wenn beide Futterquellen Lösung gleicher Konzentration enthalten, hängt die Abflugmagenfüllung nicht von der Konzentration der Lösung in der Trachtfutterquelle ab (Abb. 4).

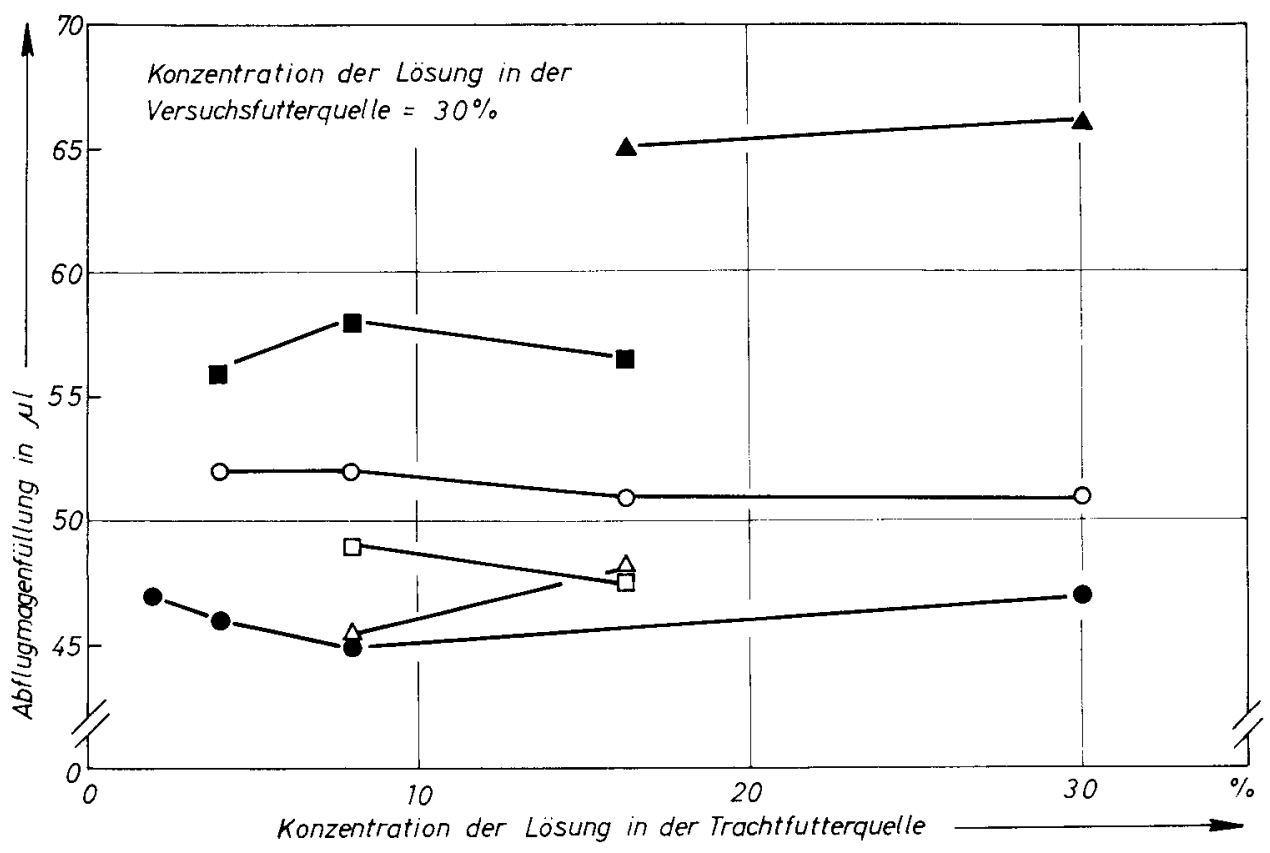

Aв8. 4. - Abflugmagenfüllung als Funktion der Konzentration der Lösung in der Trachtfutterquelle.

Konzentration der Lösung in der Versuchsfutterquelle konstant. - Jedes Symbol repräsentiert eine Biene. Zentralwerte. $4<\mathrm{n}<80$. Keine der Differenzen zwischen den Werten ist signifikant.

FIG. 4. - Remplissage du jabot en fonction de la concentration de la solution à la source de nourriture de la chambre de vol.

Concentration de la solution à la source expérimentale de nourriture constante $(30 \%)$. Chaque symbole représente une abeille. Valeurs médianes. $4<\mathrm{n}<80$. Aucune différence entre les valeurs n'est significative.

En ordonnées : remplissage du jabot en $\mu 1$.

En abscisses : concentration de la solution à la source de nourriture de la chambre de vol.

Zusätzlich zu den Versuchen, deren Ergebnisse in Abb. 4 dargestellt sind, wurden mit einer Biene Experimente durchgeführt, bei denen die Konzentration der Lösung in der Trachtfutterquelle doppelt so hoch war wie die der Lösung in der Versuchsfutterquelle. Die Werte können in Abb. 4 nicht dargestellt werden, da bei diesen Versuchen - im Unterschied zu denen der Abb. 4 - die Lösung in der Versuchsfutterquelle nicht $30 \%$ ig, sondern nur $16 \%$ ig war. Der Vergleich der bei diesen Experimenten gemessenen Abflugmagenfüllung (Zentralwert) mit der 
Abflugmagenfüllung bei Versuchen, bei denen beide Futterquellen gleich konzentrierte Lösung spendeten, liefert eine Differenz zwischen den Zentralwerten von nur $3 \mu$ l (dies sind etwa $6 \%$, wenn man auf den etwas höheren Zentralwert der Situation "Konzentration der Lösung in beiden Futterquellen gleich " bezieht). Man darf also sagen, dass auch in diesem Fall keine Abhängigkeit der Abflugmagenfüllung von den Trachtverhältnissen besteht.

Die Sauggeschwindigkeit ist bei niedrig konzentrierten Lösungen geringer als bei höheren (PFLumM, 1969 b; dies gilt wegen der Viskosität nicht für den Bereich sehr hoch konzentrierter Lösungen). Die mittlere Sauggeschwindigkeit ergibt sich als Quotient aus der Abflugmagenfüllung und der Saugdauer (das ist die für die Aufnahme dieser Menge benötigte Zeit; Näheres bei Pflumm, 1969 b). Wenn die Trachtfutterquelle niedriger konzentrierte Lösung bietet als die Versuchsfutterquelle, könnte die Biene trotz unveränderter Abflugmagenfüllung - durch schnelles Trinken anzeigen, dass die Versuchsfutterquelle relativ besser ist als die Trachtfutterquelle. Das ist nicht der Fall (Abb. 5).

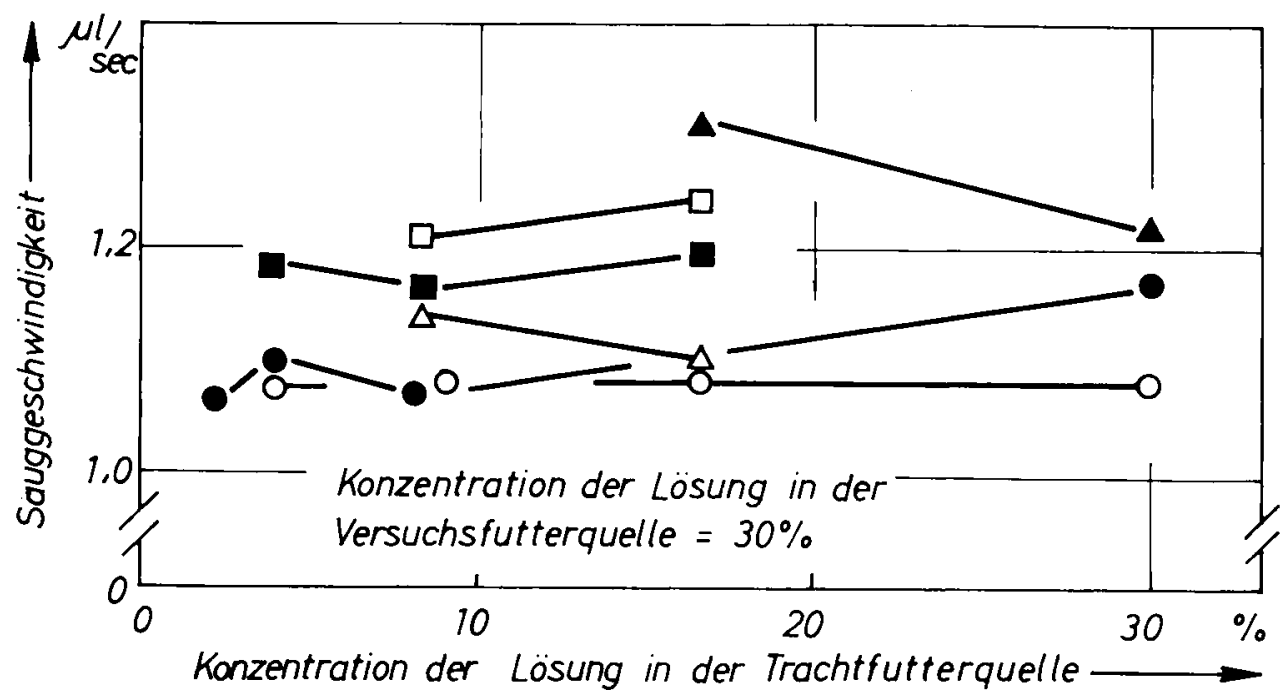

AвB. 5. - Sauggeschwindigkeit in Abhängigkeit von der Konzentration der Lösung in der Trachtfutterquelle.

Konzentration der Lösung in der Versuchsfutterquelle konstant. - Symbolik wie in Abb.4. Zentralwerte. $4<\mathrm{n}<80$. Keine Differenz ist signifikant.

FIG. 5. - Vitesse de la prise de nourriture en relation avec la concentration de la solution à la source de nourriture de la chambre de vol.

Concentration de la solution à la source expérimentale de nourriture constante (30\%).

Même symboles que pour la fig. 4 . Valeurs médianes. $4<\mathrm{n}<80$. Aucune différence n'est significative.

En ordonnées : vitesse de la prise de nourriture en $\mu \mathrm{l} / \mathrm{sec}$.

En abscisses : concentration de la solution à la source de nourriture de la chambre de vol. 


\section{DISKUSSION}

Mit Verbesserung der Trachtverhältnisse vermindert sich die Dauer des Duftmarkierens; wenn das Duftorgan an der Futterquelle überhaupt nicht ausgestülpt wird, tanzt die Sammlerin im allgemeinen im Stock nicht (v. FRISCH, 1935, 1965). Den Einfluss der Trachtverhältnisse auf den Tanz haben LiNDAUER (1948) und BOCH (1956) untersucht : LiNDAUER bot Sammelbienen von April bis September im Freien verschieden konzentrierte Lösungen und stellte jeweils fest, welche Konzentration erforderlich war, um eben noch Tänze auszulösen ("Grenzkonzentration »). Wie er fand, hängt die Grenzkonzentration von der Jahreszeit, d.h. von der Anzahl der in der Umgebung blühenden Pflanzen (blühende Obstbäume, Rapsfelder und Linden) ab. Восн registrierte die Tänze einer Bienengruppe, welche ebenfalls im Freien $12 \%$ ige Lösung an einer künstlichen Futterquelle sammelte. Als er einer zweiten Gruppe desselben Stocks, die an einer anderen künstlichen Futterquelle verkehrte, 55 \%ige Lösung bot, tanzten immer weniger Bienen der ersten Gruppe, schliesslich hörten die Tänze ganz auf.

Für die Abhängigkeit des Duftmarkierens von der Konzentration der Lösung sind sowohl die Reizsituation an der Futterquelle als auch Einflüsse im Stock massgebend. Bei der Abhängigkeit des Duftmarkierens von der Zuflussgeschwindigkeit (vgl. Abb. 8 bei Pflumm, 1969 a) dürfte allein die Reizsituation an der Futterquelle eine Rolle spielen : Die Abnehmerinnen im Stock können die Zuflussgeschwindigkeit ja nicht beurteilen.

Wenn man die Konzentration der Lösung in der Trachtfutterquelle erhöht (beispielsweise von der Situation "Lösung in beiden Futterquellen dieselbe » übergeht zur Situation " Lösung in der Trachtfutterquelle doppelt so konzentriert wie Lösung in der Versuchsfutterquelle "), kann es vorkommen, dass die Versuchsbiene nach einiger Zeit ihre Besuche an der Versuchsfutterquelle einstellt und dazu übergeht, an der Trachtfutterquelle zu sammeln. In der Zeitspanne zwischen dem Zeitpunkt des Erhöhens der Konzentration der Lösung in der Trachtfutterquelle und dem Zeitpunkt, zu dem die Versuchsbiene die Versuchsfutterquelle nicht mehr aufsucht, stülpt sie das Duftorgan nicht mehr so lange oder gar nicht mehr aus (Abb. 3), wodurch auch keine Neulinge mehr angelockt werden. Das erscheint biologisch sinnvoll. Ebenso sinnvoll ist es, dass sie während dieser Zeitspanne die gleiche Menge pro Besuch (= Abflugmagenfüllung) aufnimmt - wenn man die an der einen Biene (s. B.) gewonnenen Ergebnisse verallgemeinern darf. Die Grösse der Abflugmagenfüllung bei Besuchen, die dem Verlassen der Versuchsfutterquelle unmittelbar vorausgehen, bedarf einer weiteren Überprüfung.

Die in Abb. 1 und Einschaltfigur dargestellten Ergebnisse bestätigen die Resultate von v. Frisch (1923, 1935, 1965), eigene (PFLumm, 1969 a, 1970) und die von Nú̃̃̃ez (1971). Die Einwände von Wells und WenNer (1971), welche eine Abhängigkeit des Duftmarkierens von der Konzentration (bzw. Zuflussgeschwindigkeit) der Lösung 
bestreiten, sind somit widerlegt. Diese Autoren, welche frühere Befunde (PFLumM, 1969 a; Abb. 5) " with limited confidence " betrachten, stellen fest : " onset of scenting cannot be accurately timed because bees often open their glands while in flight prior to arriving at the feeding place ». Dies mag für den Beginn des Markierflugs gelten, jedoch nicht für die restlichen Verhaltensweisen des Duftmarkierens, deren Beginn und Ende jeweils sehr genau feststellbar ist : Sterzeln vor dem Saugen, Sterzeln während des Saugens sowie die Zeitdauer vom Saugbeginn bis zum Einstülpen des Duftorgans. Wird an der Futterquelle gesterzelt, hat zuvor Markierflug (mit ausgestülptem Duftorgan) stattgefunden (vgl. Pflumm, 1970). Die früheren (für 3 Bienen dargestellten) Daten ("... the data are sparse... ": Wells und WenNer, 1.c) werden durch die an vielen Bienen gewonnenen Resultate der Abb. 1 (und Einschaltfigur) bestätigt.

Nach der Hypothese von Wells und WENNER (1971) sollen " encounters with disoriented bees in the field lead to Nasonov gland exposure by experienced foragers at the feeding station ". Zahlreiche Begegnungen zwischen fliegenden Bienen in der Nähe der Futterquelle finden dann statt, wenn diese niedrig konzentrierte Zuckerlösung spendet. Dann unterbrechen die Bienen häufig das Saugen und fliegen in der Umgebung der Futterquelle umher; bei hohen Konzentrationen saugen sie ununterbrochen (vgl. PFLumM, 1969 a). Die umberfliegenden Sammlerinnen sind sicherlich nicht desorientiert; betrachtet man ihr Verhalten, mögen sie in starkem Masse an Suchbienen erinnern. Träfe die Hypothese von Wells und WenNER zu, müsste man ausgiebiges Duftmarkieren bei niedrigen Konzentrationen erwarten - und nicht bei hohen (Abb. 1).

Verschiedene Autoren nehmen an, dass die Bienen an künstlichen Futterquellen deshalb das Duftorgan ausstülpen, weil die Futterquellen selbst duftlos sind (FrEE, 1968; WENNER et al., 1969). Es ist möglich, dass fehlender Duft an der Futterquelle zum Auslösen des Markierverhaltens beiträgt; damit ist jedoch keine Erklärung für die unterschiedlichen Dauern des Markierens bei verschiedenen Konzentrationen gegeben.

Eine weitere Erklärung für das Duftmarkieren an künstlichen Futterquellen geben FreE und Williams (1970), welche das Duftmarkieren von Wassersammlerinnen untersuchten. Nach diesen Autoren ist Duftmarkieren " probably a response to an abundant supply of forage that is usually associated only with foraging for water . Wenn Duftmarkieren eine Reaktion auf " an abundant supply of forage " wäre, müsste man es auch bei zwar niedrig konzentriertem, jedoch reichlich fliessendem Zuckerwasser beobachten - was nicht der Fall ist (Abb. 1).

Eigene Beobachtungen an Wassersammlerinnen im Freien stützen die Angabe von FreE und Williams (1970) nicht: Am 8., 14., 15. und 26. Juni 1970 wurde das Verhalten von Bienen, welche etwa $10 \mathrm{~m}$ vom Stock entfernt Wasser sammelten, registriert (10-20 Bienen; Luftemperatur 17-20 ${ }^{\circ} \mathrm{C}$ ). Sie saugten klares Quellwasser (Temperatur $10^{\circ} \mathrm{C}$ ), wobei sie solche Stellen bevorzugten, wo Steine von einem Wasserfilm überzogen waren. Nur bei 3 von 210 Besuchen war das Duftorgan ausgestülpt. 


\title{
DANK
}

Herrn Imkermeister DrucK danken wir für Hilfe bei der Bienenhaltung, Herrn Dr. D. GraHAM für Korrektur der englischen Zusammenfassung.

\author{
Eingegangen im Juni 1978. \\ Reçu pour publication en juin 1978.
}

\section{RÉSUMÉ}

Dans une chambre de vol on a reproduit des conditions de miellée qui offraient aux butineuses (Apis mellifica) des solutions de saccharose à diverses concentrations. Ces solutions étaient données à une " source de nourriture de la chambre de vol n. A une deuxième source de nourriture (source expérimentale de nourriture) la concentration de la solution de saccharose était maintenue constante. Là on introduisait une seule abeille à la fois et enregistrait son comportement en liaison avec la concentration de la solution à la source de nourriture de la chambre de vol.

On entend par conditions de miellée la production de nectar de toutes les sources de nourriture qui sont visitées par les abeilles d'une même colonie.

Les expériences ont duré d'août à novembre 1975 et 1976 et ont porté sur des colonies de race carnolienne. Jusqu'au début de la période d'acclimatation, d'une durée d'une semaine, la ruche est à l'extérieur. Dans la chambre de vol (selon Renner, 1955, et VAN PraAgh, 1974) règne la température de $23 \pm 3^{\circ} \mathrm{C}$. L'humidité relative varie entre 50 et $60 \%$. L'eau est fournie ad libitum et le pollen frais (pelotes broyées) changé toutes les heures et quart.

Un nourrisseur (pneumatique), situé à $3 \mathrm{~m}$ de la ruche, dont le bord inférieur est entouré d'un sillon de $7 \mathrm{~mm}$ de large, contient l'eau sucrée sur une hauteur d'environ $5 \mathrm{~mm}$; de cette façon " l'approvisionnement total " en solution est assuré. Ce nourrisseur est désigné dans ce qui suit par l'expression a source de nourriture de la chambre de vol $\mathrm{y}$.

La source expérimentale de nourriture (fleur artificielle, voir fig. 1 dans PflumM, 1977) se trouve à un mètre de la source de nourriture de la chambre de vol, dans une pièce contiguë (température $24 \pm 1,5^{\circ} \mathrm{C}$ ), à laquelle les butineuses ont accès par une ouverture de $40 \times 40 \mathrm{~cm}$. Là on expérimente avec des abeilles individuelles; les novices sont refoulées.

La succession des solutions de saccharose $\mathrm{D}-(+)$ de concentration variable se fait suivant une suite statistique aléatoire (concentrations données en \%, c'est-à-dire en grammes pour $100 \mathrm{~g}$ de solution; abréviation pour concentration : c) Lorsqu'on change la concentration de la solution dans la source de nourriture de la chambre de vol (c'est-à-dire quand les " conditions de miellée "varient), la concentration de la solution dans la source expérimentale de nourriture est maintenue constante et inversement.

Lors d'une expérience spéciale (voir A) des mesures ont été faites sur des abeilles qui fréquentaient la source expérimentale de nourriture.

Dans toutes les expériences il y a approvisionnement total en solution, c'est-à-dire que les abeilles peuvent boire aussi vite qu'elles le désirent.

Le remplissage du jabot est mesuré de la façon suivante : la solution sucrée afflue vers la butineuse dans une pipette graduée à l'intérieur de laquelle une vésicule remplie d'air indique par son déplacement la quantité absorbée.

La vitesse moyenne de prise de nourriture est le quotient du contenu du jabot par le temps nécessaire pour prélever cette quantité.

Lorsque la concentration de la solution dans la source de nourriture de la chambre de vol croît (c'està-dire lorsque les conditions de miellée s'améliorent), la durée du marquage olfactif pendant la prise de nourriture décroît (fig. 3). Si la source de nourriture de la chambre de vol fournit une solution moins concentrée que celle de la source expérimentale de nourriture ou si la concentration de la solution est la 
même dans les deux sources de nourriture, le remplissage du jabot et la vitesse de la prise de nourriture ne sont pas influencés par les conditions de miellée (fig. 4).

Lorsque les conditions de miellée sont maintenues constantes et que la concentration de la solution dans la source expérimentale de nourriture varie, les mesures du comportement faites dans la chambre de vol sont semblables à celles obtenues à l'extérieur (fig. 1).

Les expériences à l'extérieur (résultats dans la figure encadrée de la fig. 1) ont été menées durant la seconde moitié de juillet 1974 à la température de $19,0 \pm 1,5^{\circ} \mathrm{C}$ avec un groupe de 20 à 30 abeilles à une distance de $6 \mathrm{~m}$ de la ruche. Un récipient en verre largement ouvert (petite boite de Pétri) utilisé comme source de nourriture garantit l'approvisionnement total pour toutes les butineuses. Puisqu'on ne peut observer en même temps toutes les butineuses en train de boire une coupelle, on a procédé de la façon suivante : pour une abeille donnée on a enregistré le temps entre le début de la prise de nourriture et le moment où la glande de Nasonov était rentrée; la mesure suivante était prise chez l'abeille qui atterrissait le plus près.

Les résultats concernant le marauage olfactif confirment ceux de v. FRISCH $(1923,1935,1965)$ les nôtres (PFLumm, 1969 a et 1970) et ceux de NúÑEz (1971). Les résultats sont confrontis avec les donneses contradictoires d'autres auteurs (WELLs et WENNER, 1971) et leurs hypothèses (FrEE, 1968; FREE et WILLIAMS, 1970; WENNER et al. 1969). Les abeilles qui récoltent de l'eau à l'extérieur n'exposent généralement pas leur glande de Nassonov (on n'a observé la dévagination que dans $1,4 \%$ des visites).

\section{LITERATUR}

Boch R., 1956. - Die Tänze der Bienen bei nahen und fernen Trachtquellen. Z. vergl. Physiol., 38, 136167.

FrEE J. B., 1968. - The conditions under which foraging honeybees expose their Nasonov gland. J. apic. Res., 7, 139-145.

Free J. B. and Williams Ingrid H., 1970. - Exposure of the Nasonov gland by honeybees (Apis mellifera) collecting water. Behaviour, 37, 286-290.

Frisch K. v., 1923. - Über die "Sprache" der Bienen, eine tierpsychologische Untersuchung. Zool. Jb., Abt. allg. Zool. u. Physiol., 40, 1-186.

FrISCH K. v., 1935. - Uber den Geschmackssinn der Bienen. Z. vergl. Physiol., 21, 1-157.

FrISCH K., v., 1965. - Tanzsprache und Orientierung der Bienen. Springer, Berlin-Heidelberg-New York, $578 \mathrm{~S}$.

Lindauer M., 1948. - Utber die Einwirkung von Duft- und Geschmacksstoffen sowie anderer Faktoren auf die Tänze der Bienen. Z. vergl. Physiol., 31, 348-412.

NúÑ̃z J. A., 1971. - Beobachtungen an sozialbezogenen Verhaltensweisen von Sammelbienen. $Z$. Tierpsychol., 28, 1-18.

PFlumm W., 1969 a. - Beziehungen zwischen Putzverhalten und Sammelbereitschaft bei der Honigbiene. Z. vergl. Physiol., 64, 1-36.

PfLumm W., 1969 b. - Stimmungsänderungen der Biene während des Aufenthalts an der Futterquelle. $Z$. vergl. Physiol., 65, 299-323.

Pflumm W., 1970. - Auslösende Faktoren beim Duftmarkieren von Sammelbienen. Experientia (Basel), 26, 318-319.

Pflumm W., 1977. - Welche Grössen beeinflussen die Menge der von Bienen und Wespen an der Futterquelle aufgenommenen Zuckerlösung. Apidologie, 8, 401-412.

PraAgh J. P. van de., 1974. - De ontwikkeling van een klimaatkamer voor de honingbij. Dissertation, Rijksuniversiteit te Utrecht.

RENNER M., 1955. - Über die Haltung von Bienen in geschlossenen, künstlich beleuchteten Räumen. Naturwissenschaften, 42, 539-540. 
RenNer M., 1959. - Über ein weiteres Versetzungsexperiment zur Analyse des Zeitsinnes und der Sonnenorientierung der Honigbiene. Z. vergl. Physiol., 42, 449-483.

RenNer M., 1960. - Das Duftorgan der Honigbiene und die physiologische Bedeutung ihres Lockstoffes. Z. vergl. Physiol., 43, 411-468.

RibbandS C. R., 1955. - Communication between honeybees : II. The recruitment of trained bees, and their response to improvement of the crop. Proc. Roy. Ent. Soc. Lond., (A), 30, 1-3.

RUtTNer F. und Koeniger N. (redig.) 1977. - Bienenhaltung in Flugräumen; Symposium über Flugraumtechnik. Insectes sociaux, 24, 279-302.

Wells P. H. and WENNER A. M., 1971. - The influence of food scent on behavior of foraging honey bees. Physiol. Zool., 44, 191-209.

Wenner A. M., Wells P. H. and Johnson D. L., 1969. - Honey bee recruitment to food sources : olfaction or language? Science, $\mathbf{1 6 4}, 84-86$. 